\title{
Sensor for real-time animal condition and movement monitoring *
}

\author{
Altynay Kaidarova ${ }^{1}$, Muhammad Akram Karimi ${ }^{1}$, Selma Amara ${ }^{1}$, Atif Shamim ${ }^{1}$, Nathan R. Gerali ${ }^{2}$, Carlos M. \\ Duarte $^{2}$ and Jurgen Kosel ${ }^{1}$.
}

${ }^{1}$ Computer, Electrical and Mathematical Sciences \& Engineering (CEMSE), King Abdullah University of Science and Technology(KAUST), Thuwal, Kingdom of Saudi Arabia

${ }^{2}$ Red Sea Research Center (RSRC), King Abdullah University of Science and Technology (KAUST)

Kingdom of Saudi Arabia, Thuwal 23955

jurgen.kosel@kaust.edu.sa

\begin{abstract}
A flexible, lightweight and minimally intrusive monitoring system has been developed to assess animals' behavioral responses. The system consists of wearable composite magnets and magnetic sensors integrated into a miniaturized wireless communication module with a flexible battery. The shape and size of the NdFeB-PDMS composite magnets are highly versatile, while the magnetic and mechanical properties can be tailored within a wide range by the powder concentration. The magnetic field of the composite magnet is sensed by a 3 -axial magnetic sensor, and the measured data is wirelessly transmitted using Bluetooth low energy communication standard to a smartphone and dashboard. To withstand corrosive environments and enhance the durability the composite magnets are coated with $2 \mu \mathrm{m}$ of Parylene $\mathrm{C}$, while surface passivation of the wireless module is achieved with $5 \mu \mathrm{m}$ of Parylene C. The system has been implemented for real-time monitoring of crabs, giant turtles, and giant clams, indicating its potential for novel and affordable animal monitoring applications.
\end{abstract}

\section{INTRODUCTION}

Understanding the behavior of animal species is a necessary underpinning of conservation efforts and to assess how multiple pressures, such as climate change, may affect marine ecosystems [1]. Hence, biologging devices to acquire quantitative datasets on animals' behaviors have been developed [2],[3]. Yet, they use heavy, bulky and mechanically inflexible systems and satellite-linked data transference systems on large species, with perceptible impacts on their undisturbed behavior [4].

A promising solution along this line utilizes magnetic field sensors and small permanent magnets to examine animal movements. Such magnetic sensor systems are usually composed of neodymium magnets and a magnetic field sensor. A high tolerance of animals to magnetic fields [5] and the measurable magnetic properties exhibited by magnets in air and water [6] makes this approach particularly appealing to overcome the limitations of existing biologging devices.

Here, we aim to improve animals' comfort and its free movement while their activity is being logged by developing a flexible, lightweight and minimally intrusive monitoring system, composed of $\mathrm{NdFeB/PDMS}$ composite magnets, magnetic sensors and a miniaturized wireless data acquisition unit. The proposed system enables simple and reliable means to reveal novel information about animals, such as daily activity, eating behavior, pregnancy, and breathing behavior. These could be readily achieved by monitoring the body movement of animals, such as limbs movement, heart rate, mouth movement, changes in belly size and/or gill movements. In its ultimate form, different types of movements will be collected and analyzed to obtain specific information as shown in Fig.1.

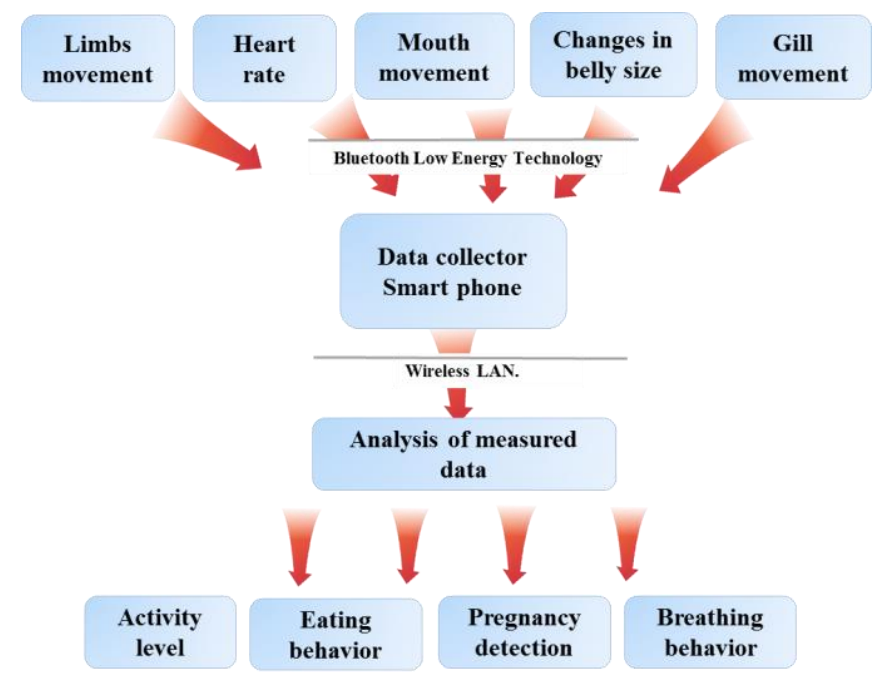

Fig. 1. The data flow of the animal montoring system. The body movements that are being monitored are examples among many possibilities.

\section{SYSTEM CONFIGURATION}

\section{A. System Concept}

The magnetic sensor system is based on the detection of the magnetic field emanating from the composite magnet by a magnetic sensor. The magnet is attached to the animal's moving body part, such as a fin, that varies its position with 
respect to another body part, such as the head, on which a magnetic field sensor is mounted. The movement of the fin with respect to the head causes a variation in the magnetic field intensity recorded by the magnetic sensor. The wireless monitoring system acquires the signals from the sensor, transmits them via a Bluetooth link, displays and stores the measured data on a smart-phone and computer, as shown in Fig. 2.

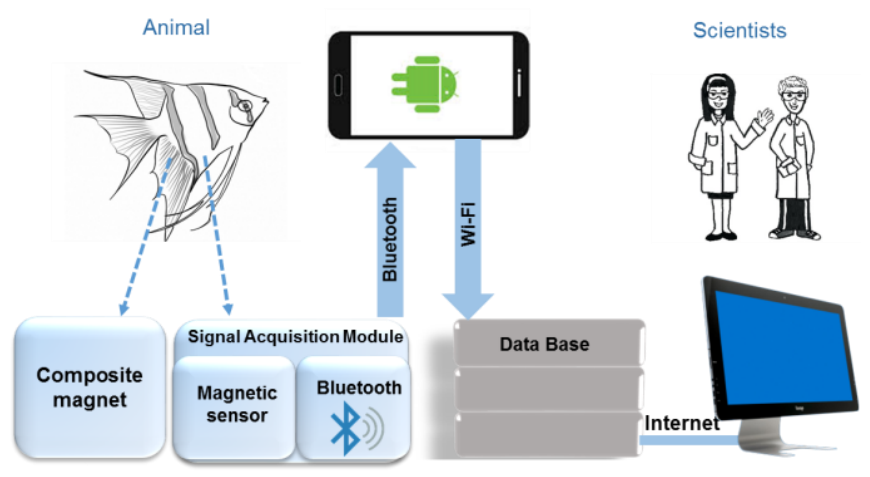

Fig. 2. Schematic of real-time marine animals' monitoring system.

\section{B. NdFeB/PDMS composite magnets}

Composite magnets were fabricated, as shown in Fig.3, by firstly preparing the polydimethylsiloxane (PDMS, Dow Corning Corp. Slygard $®$ 184) through mixing the elastomer and the curing agent using a 10:1 weight ratio. $\mathrm{NdFeB}$ microparticles (Molycorp MQP-16-7FP, $5 \mu \mathrm{m}$ ) were then added by mechanical stirring and the composite poured into polymethylmethacrylate (PMMA) molds fabricated using a $\mathrm{CO} 2$ laser cutter (Universal Laser Systems Inc.PLS 6.75). Depending on the design of the PMMA molds, various customized elastic shapes and sizes can be obtained as shown in Fig.4. The magnetic particles are then aligned with a magnetic field prior to curing the PDMS, which was recently shown to increase the remanent magnetization by $\sim 16 \%$ [5] and attributed to the alignment of an anisotropy axis with the field direction. Thus, a unidirectional magnetic field of $1.5 \mathrm{~T}$ was applied before curing at $90^{\circ} \mathrm{C}$ for an hour. Carefully demolded composite magnets are also coated with Parylene $\mathrm{C}$ film of $2 \mu \mathrm{m}$ thickness to enhance durability and simultaneously obtain corrosion resistance, and increased biocompatibility [5].

To investigate the effect of powder concentrations on the elasticity and magnetic properties, three composite samples with differing $\mathrm{NdFeB}$ concentrations (10\% wt, 50\% wt and $70 \% \mathrm{wt})$ were fabricated. The results in Fig. 5 indicate that the remanent and saturation magnetization of the magnetic composites have a positive linear relationship with the filler concentration, as expected.

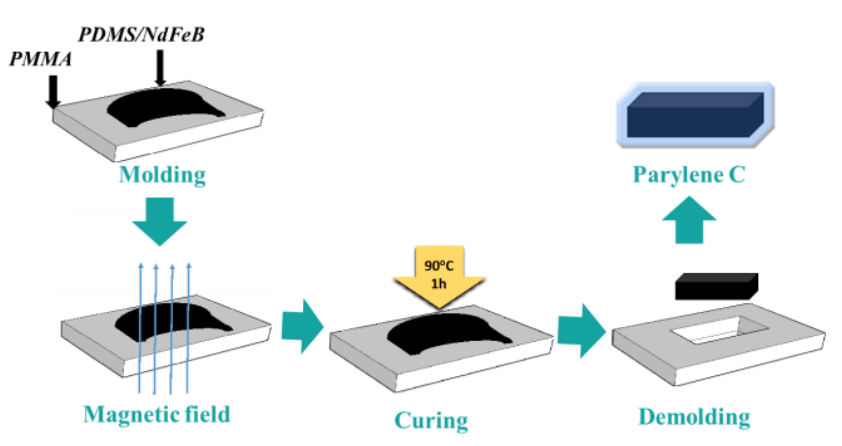

Fig. 3. Fabrication process of NdFeB/PDMS composite magnets.

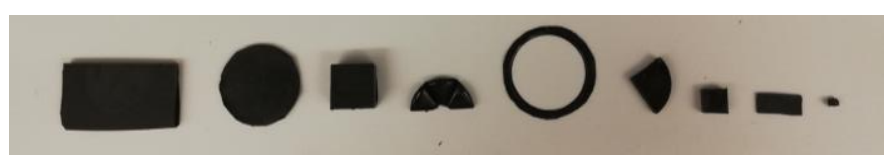

Fig.4. Samples of various shapes and sizes of composite magnets.

The tensile test, shown in Fig. 6, revealed that the Young's modulus also increases with powder content, due to the reduced volume of the polymer bearing the stress in the powder network. The Young's modulus of the composite magnets is almost $10^{5}$ times less than that of commercially available permanent magnets [6]. A significant weight reduction is also achieved, i.e. composite magnets of $50 \% \mathrm{wt}$ and $10 \% \mathrm{wt}$ are 3 times and 13 times lower in weight, respectively. This is critically important, since the decreased weight of the magnetic composites imposes less burden on animals.

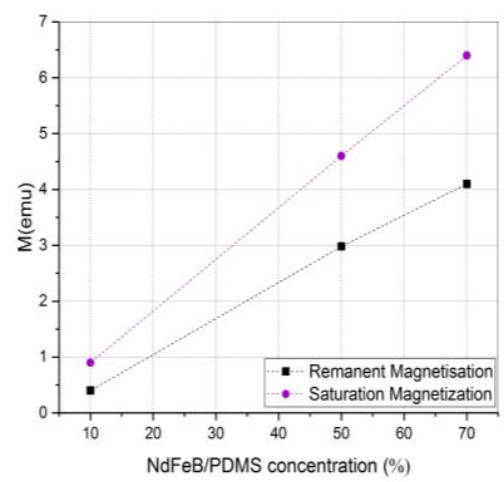

Fig. 5. a) Relationship between magnetisations of composite magnet and powder concentration.

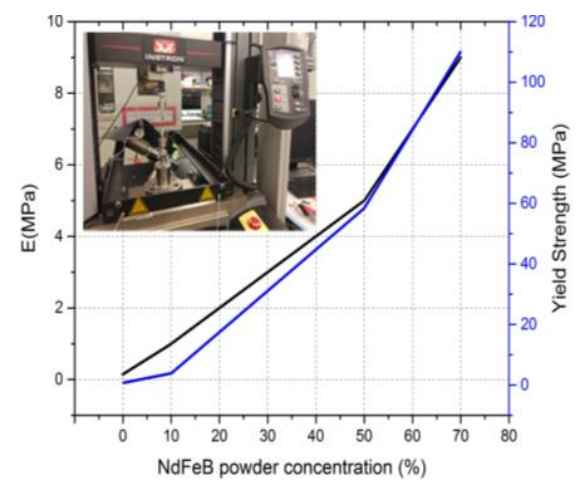

Fig. 6. Modulus of elasticity and yield strength as a function of $\mathrm{NdFeB}$ powder concentration. Insets show the measurement system and the flexibility of the composite magnet. 


\section{Signal Acquisition Module}

A three-axial magnetic tunnel junction sensor (TMR2305, Multi Dimension, Inc.) has been utilized to measure the magnetic stray field of the flexible NdFeB-PDMS composite magnets. The sensor has been combined with a Bluetooth chip (nRF52832, Nordic Semiconductor) through a I2C communication interface on a miniaturized PCB and flexible battery as shown in Fig. 7a and Fig. 7b. The PCB was coated with $5 \mu \mathrm{m}$ biocompatible and waterproof Parylene C.
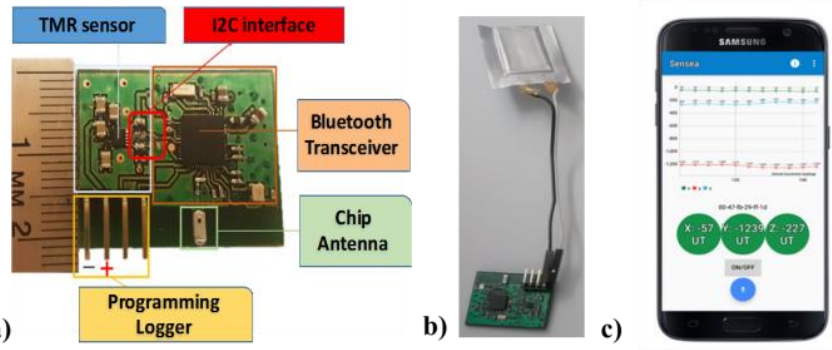

Fig. 7. a) Components of the miniaturized PCB. b) Assembled system with flexible battery. c) Smart phone application visualizing the vectors of the magnetic field.

The Bluetooth chip is utilized to wirelessly transmit the sensor readings to a Bluetooth enabled smart device. The three vectors of the magnetic field are sent over Bluetooth protocol at a refresh rate of 10 readings per second. An Android-based smart phone application was developed to visualize the magnetic field in the form of its 3 vectors, as shown in Fig. 7c. The application searches for those Bluetooth devices, whose service UUID (Universal Unique Identifier) corresponds with the one attributed to our customized sensor service. The data was transmitted to up to 100 meters. The measured data is then transmitted to a dashboard server and downloaded to a computer for further processing and analysis.

\section{ANIMAL MONITORING}

The system was implemented for real-time monitoring of crabs (Libinia emarginata), giant turtles (Aldabrachelys gigantean) and giant clam (Tridacna gigas). To assess the physical activity level of crabs and turtles, the sensor was attached on the carapaces and the magnet on their legs, as shown in Fig. 8a and 8b. Based on the obtained signals, steps of three different magnitudes are identified for the crab, while the active and resting periods of the giant turtle is readily identified as well.

The signals derived from a giant clam's monitoring experiment enable extracting essential information, such as levels of activity over time (e.g. day time versus night) or as a function of proximity to predators, as illustrated in Fig. 9. No indication of deterioration of the magnets or sensors were noted during the experiments, which lasted up to one day.
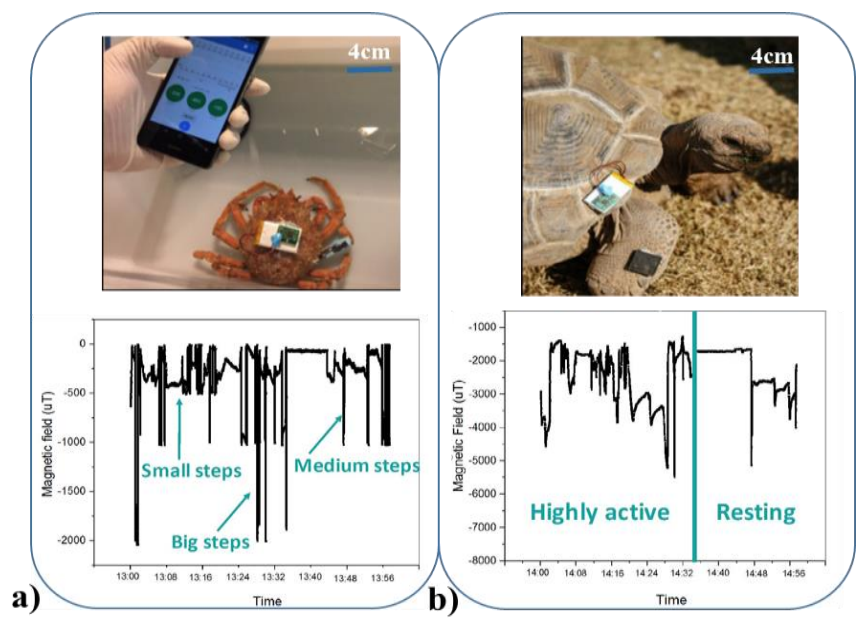

Fig. 8. Monitoring of the leg activity of a) crab and b) giant turtle.
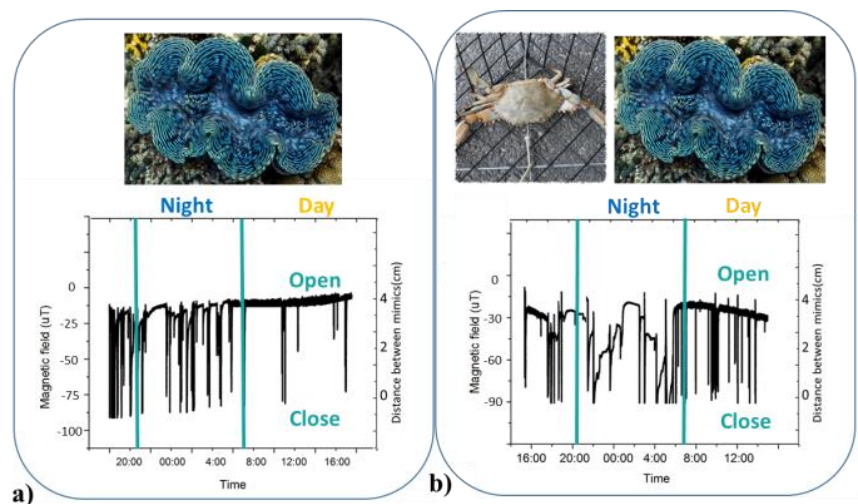

Fig. 9. a) Investigation of activity level of giant clam during day versus night time and b) effect of external disturbances, such as predator, to its natural behavior

\section{CONCLUSION}

The developed animal monitoring system provides a simple and cost-effective method for animal studies. The tailored magnets provide a high degree of customization, which can be exploited to minimize the impact on the monitored animals as well as optimize the measured signals. The experimental results suggest the suitability of the proposed system for monitoring applications of a wide range of animals. Due to the miniaturized PCB and versatile composite magnets the opportunities have also vastly expanded to include tagging of small animals that could previously not be monitored, due to the size, weight and rigidity of existing systems. Combining this system with satellite trackers in the next stage, will allow monitoring of animals freely moving across ocean basins.
This research is a contribution to the CAASE project funded by King Abdullah University of Science and Technology (KAUST) under the KAUST Sensor Initiative. We thank the Oceanografic, Valencia, and their staff for invaluable help while conducting this research. 


\section{REFERENCES}

[1] Cooke SJ, Hinch SG, Wikelski M, Andrews RD, Kuchel LJ, Wolcott TG, Butler PJ. Biotelemetry: a mechanistic approach to ecology. Trends in ecology \& evolution. 2004 Jun 1;19(6):334-43.

[2] Block BA, Costa DP, Boehlert GW, Kochevar RE. Revealing pelagic habitat use: the tagging of Pacific pelagics program. Oceanologica Acta. 2002 Sep 1;25(5):255-66.

[3] Lander ME, Lindstrom T, Rutishauser M, Franzheim A, Holland M. Development and field testing a satellite-linked fluorometer for marine vertebrates. Animal Biotelemetry. 2015 Dec;3(1):40.
[4] Franklin PA, Baker CF. Fish passage research in the Southern Hemisphere: Challenges, lessons and the need for novel solutions. In International Symposium on Ecohydraulics, Melbourne, Australia 2016.

[5] Kaidarova A, Khan MA, Amara S, Geraldi NR, Karimi MA, Shamim A, Wilson RP, Duarte CM, Kosel J. Tunable, Flexible Composite Magnets for Marine Monitoring Applications. Advanced Engineering Materials. 2018.

[6] Rabinovich YM, Sergeev VV, Maystrenko AD, Kulakovsky V, Szymura $\mathrm{S}$, Bala $\mathrm{H}$. Physical and mechanical properties of sintered $\mathrm{Nd} \cdot \mathrm{Fe} \cdot \mathrm{B}$ type permanent magnets. Intermetallics. 1996 Jan 1;4(8):641-5. 